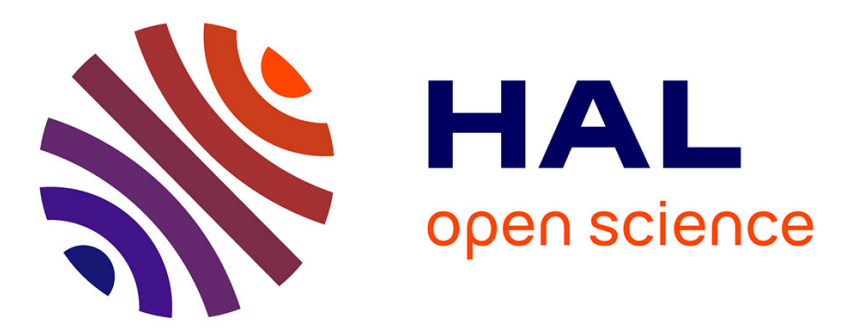

\title{
New Method for Global Identification of the Joint Drive Gains of Robots using a Known Payload Mass
}

\author{
Maxime Gautier, Sébastien Briot
}

\section{To cite this version:}

Maxime Gautier, Sébastien Briot. New Method for Global Identification of the Joint Drive Gains of Robots using a Known Payload Mass. IEEE/RSJ International Conference on Intelligent Robots and Systems (IROS 2011), Sep 2011, San Francisco, France. hal-00609073

\section{HAL Id: hal-00609073 https://hal.science/hal-00609073}

Submitted on 25 Jun 2019

HAL is a multi-disciplinary open access archive for the deposit and dissemination of scientific research documents, whether they are published or not. The documents may come from teaching and research institutions in France or abroad, or from public or private research centers.
L'archive ouverte pluridisciplinaire HAL, est destinée au dépôt et à la diffusion de documents scientifiques de niveau recherche, publiés ou non, émanant des établissements d'enseignement et de recherche français ou étrangers, des laboratoires publics ou privés. 


\title{
New Method for Global Identification of the Joint Drive Gains of Robots using a Known Payload Mass
}

\author{
Maxime Gautier and Sébastien Briot
}

\begin{abstract}
Off-line robot dynamic identification methods are mostly based on the use of the Inverse Dynamic Identification Model (IDIM), which calculates the joint force/torque that is linear in relation to the dynamic parameters, and on the use of linear least squares technique to calculate the parameters (IDIM-LS technique). The joint forces/torques are calculated as the product of the known control signal (the current reference) by the joint drive gains. Then it is essential to get accurate values of joint drive gains to get accurate identification of inertial parameters. In this paper it is proposed a new method for the identification of the total joint drive gains in one step using available joint sampled data given by the standard controller of the moving robot and using only the weighted mass of a payload, without any CAD values of its inertial parameters. A new inverse dynamic model calculates the current reference signal of each joint $\mathbf{j}$ that is linear in relation to the dynamic parameters of the robot, to the inertial parameters of a known mass fixed to the end-effector, and to the inverse of the joint $j$ drive gain. This model is calculated with current reference and position sampled data while the robot is tracking one reference trajectory without load on the robot and one trajectory with the known mass fixed on the robot. Each joint $j$ drive gain is calculated independently by the weighted LS solution of an over-determined linear systems obtained with the equations of the joint $\mathrm{j}$. The method is experimentally validated on an industrial Stäubli $\mathbf{R X}-90$ robot.
\end{abstract}

\section{INTRODUCTION}

$\mathrm{S}^{\mathrm{E}}$ EVERAL schemes have been proposed in the literature to identify the dynamic parameters of robots [1]-[8]. Most of the dynamic identification methods have the following common features:

- the use of an Inverse Dynamic Identification Model (IDIM) which calculates the joint force/torque linear in relation to the dynamic parameters,

- the construction of an over-determined linear system of equations obtained by sampling IDIM while the robot is tracking some trajectories in closed-loop control,

- the estimation of the parameter values using least squares techniques (LS).

This procedure is called the IDIM-LS technique.

The experimental works have been carried out either on prototypes in laboratories or on industrial robots and have

Manuscript received March 15, 2011.

M. Gautier is with the Institut de Recherche en Communication et Cybernétique de Nantes (IRCCyN) and with the University of Nantes, 44321 Nantes France (phone: +33(0)240376960; fax: +33(0)240376930; email: Maxime.Gautier@irccyn.ec-nantes.fr).

S. Briot is with the Institut de Recherche en Communication et Cybernétique de Nantes (IRCCyN), 44321 Nantes France (e-mail: Sebastien.Briot@irccyn.ec-nantes.fr). shown the benefits in terms of accuracy in many cases. Good results can be obtained provided two main conditions are satisfied:

- a well-tuned derivative band-pass filtering of joint position is used to calculate the joint velocities and accelerations,

- the accurate values of joint drive gains $g_{\tau}$ are known to calculate the joint force/torque as the product of the known control signal calculated by the numerical controller of the robot (the current references) by the joint drive gains [9].

This needs to calibrate the drive train constituted by a current controlled voltage source amplifier with gain $G_{i}$ which supplies a permanent magnet DC or a brushless motor with torque constant $K_{t}$ coupled to the link through direct or gear train with gear ratio $N$.

Because of large values of the gear ratio for industrial robots, $(N>50)$, joint drive gain, $g_{\tau}=N G_{i} K_{t}$, is very sensitive to errors in $G_{i}$ and $K_{t}$ which must be accurately measured from special, time consuming, heavy tests, on the drive chain [9].

In this paper it is proposed a new method for the identification of the total joint drive gains in one step, using current reference and position sampled data while the robot is tracking one reference trajectory without load fixed on the robot and one trajectory with a known mass fixed on the robot whose inertial parameters are measured 1. Each joint $j$ drive gain is calculated independently by the weighted LS solution of an over-determined linear system obtained with the equations of the joint $j$.

The method is experimentally validated on an industrial Stäubli RX-90 robot.

The paper is organized as follows: section 2 recalls the dynamic modelling and identification procedures. Section 3 deals with the new modelling and identification method for the robot drive gains parameters. Section 4 presents the experimental validations. Finally, section 5 gives the conclusion.

\section{USUAL INVERSE DYNAMIC MODELS AND IDENTIFICATION}

\section{A. Dynamic Modelling}

In the following, all mechanical variables are given in SI units on the joint side. All forces/torques, positions, velocities and accelerations have a conventional positive sign 
in the same direction. That defines a motor convention for the mechanical behaviour.

The inverse dynamic model (IDM) of a rigid robot composed of $n$ moving links calculates the motor force/torque vector, as a function of the generalized coordinates and their derivatives. It can be obtained from the Newton-Euler or the Lagrangian equations [6]:

$\tau_{d y n}=\tau_{i n}+\tau_{f}$

where:

$\tau_{d y n}$ is the $(n \times 1)$ vector of dynamic forces/torques due to the inertial, centrifugal, Coriolis, and gravitational effects:

$\tau_{d y n}=M(q) \ddot{q}+C(q, \dot{q}) \dot{q}+Q(q)$

$q, \dot{q}, \ddot{q}$, are respectively the $(n \times 1)$ vectors of generalized joint positions, velocities and accelerations,

$M(q)$ is the $(n \times n)$ robot inertia matrix,

$C(q, \dot{q})$ is the $(n \times n)$ matrix of centrifugal and Coriolis effects,

$Q(q)$ is the $(n \times 1)$ vector of gravitational forces/torques.

$\tau_{i n}$ is the $(n \times 1)$ input electromagnetic force/torque vector of the drive chain, in SI units on the joint side.

$\tau_{\text {in }}=g_{\tau}\left(v_{\tau i d m}-v_{\tau 0}\right)$

where:

$v_{\text {tidm }}$ is the $(n \times 1)$ vector of current references of the current amplifiers,

$v_{\tau 0}$ is the $(n \times 1)$ vector of amplifier offsets,

$g_{\tau}$ is the $(n \times n)$ matrix of the drive gains,

$g_{\tau}=N G_{i} K_{t}$

$N$ is the $(n \times n)$ gear ratios matrix of the joint drive chains such as, $\dot{q}_{m}=N \dot{q}$, with $\dot{q}_{m}$ the $(n \times 1)$ vector of motor velocities on the motor side,

$G_{i}$ is the $(n \times n)$ static gains diagonal matrix of the current amplifiers, assuming a large current loop bandwidth, usually close to $1 \mathrm{KHz}$.

$K_{t}$ is the $(n \times n)$ diagonal matrix of the electromagnetic motor torque constants.

It should be mentioned that the components of matrices $N, G_{i}$ and $K_{t}$ are data that are generally given by the manufacturers, but with a quite important inaccuracy. They can also be independently identified using the methods presented in [9]. However, these methods involve special tests on the joint drive train of the robot which are heavy and time consuming procedures.

$\tau_{f}$ is the $(n \times 1)$ vector of the loss force/torque due to frictions and motor iron losses, eddy currents and hysteresis effect. Usually, it is approximated with a viscous friction coefficient and a Coulomb friction force/torque:

$\tau_{f}=-F_{v} \dot{q}-F_{c} \operatorname{sign}(\dot{q})-F_{\text {coff }}$

where:

$F_{v}$ is the $(n \times n)$ diagonal matrix of viscous parameters,
$F_{c}$ is the $(n \times n)$ diagonal matrix of dry friction parameters, and $\operatorname{sign}($.$) denotes the sign function,$

$F_{\text {coff }}$ is a $(n \times 1)$ vector of asymmetrical Coulomb friction force/torque between positive and negative velocities.

Thus (1) becomes:

$\tau_{d y n}=\tau_{i d m}-F_{v} \dot{q}-F_{c} \operatorname{sign}(\dot{q})-\tau_{o f f}$

where:

$\tau_{\text {off }}=F_{\text {coff }}+g_{\tau} v_{\tau 0}$ is the $(n \times 1)$ vector of offset force/torque that regroups the amplifier offset and the asymmetrical Coulomb friction coefficient.

$\tau_{i d m}=g_{\tau} v_{\text {tidm }}$ defines the motor force/torque which includes the amplifier offset component.

Then the inverse dynamic model (IDM) is given by:

$\tau_{i d m}=M(q) \ddot{q}+N(q, \dot{q})$

$N(q, \dot{q})$ regroups the $(n \times 1)$ vector of centrifugal, Coriolis, gravitational, friction and offset forces/torques:

$N(q, \dot{q})=C(q, \dot{q}) \dot{q}+Q(q)+F_{v} \dot{q}+F_{c} \operatorname{sign}(\dot{q})+\tau_{o f f}$

\section{B. Identification Model}

The modified Denavit and Hartenberg notation allows obtaining a dynamic model (7) that is linear in relation to a set of standard dynamic parameters, $\chi_{s t}$ :

$\tau_{i d m}\left(q, \dot{q}, \ddot{q}, \chi_{s t}\right)=\Phi_{s t}(q, \dot{q}, \ddot{q}) \chi_{s t}$

where:

$\Phi_{s t}(q, \dot{q}, \ddot{q})$ is the $\left(n \times n_{s t}\right)$ jacobian matrix of $\tau_{i d m}$, with respect to the $\left(n_{s t} \times 1\right)$ vector $\chi_{s t}$ of the standard parameters given by $\chi_{s t}=\left[\begin{array}{llll}\chi_{s t}^{1 T} & \chi_{s t}^{2 T} & \cdots & \chi_{s t}^{n T}\end{array}\right]^{T}$ :

$\chi_{s t}^{j}$ is the $(14 \times 1)$ vector of standard dynamic parameters of joint and link $j$ :

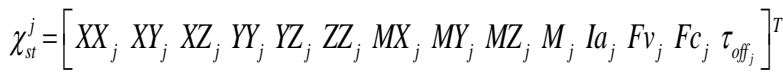

where:

$X X_{j}, X Y_{j}, X Z_{j}, Y Y_{j}, Y Z_{j}, Z Z_{j}$ are the 6 components of the inertia matrix of link $j$ at the origin of frame $j$.

$M X_{j}, M Y_{j}, M Z_{j}$ are the 3 components of the first moment of link $j$.

$M_{j}$ is the mass of link $j$,

$I a_{j}$ is a total inertia moment for rotor and gears of actuator $j$.

$F v_{j}, F c_{j}, \tau_{o f f_{j}}$ are the coefficients of the $F_{v}, F_{c}, \tau_{o f f}$, matrices, respectively.

The columns $\Phi_{s t}(:, i)$ of the matrix $\Phi_{s t}(q, \dot{q}, \ddot{q})$ can be obtained using the recursive algorithm of Newton-Euler, which calculates $\tau_{i d m}$ (7) in terms of the standard dynamic parameters, such that:

$\Phi_{s t}(:, i)=\tau_{i d m}\left(q, \dot{q}, \ddot{q}\right.$, with $\chi_{s t_{i}}=1, \chi_{s t_{k}}=0$, for $\left.k \neq i\right)$ 
To increase the efficiency of this algorithm, we use the customized symbolic technique [6], [14].

The identifiable parameters are the base parameters which are the minimum number of dynamic parameters from which the dynamic model can be calculated. They are obtained from the standard inertial parameters by regrouping some of them by means of linear relations [15], which can be determined for the serial robots using simple closed-form rules [3], [6], or by numerical method based on the QR decomposition [13].

The minimal dynamic model can be written using the $n_{b}$ base dynamic parameters $\chi$ as follows:

$\tau_{i d m}=\Phi(q, \dot{q}, \ddot{q}) \chi$

where $\Phi$ is obtained from $\Phi_{s t}$ by eliminating the columns corresponding to the non identifiable parameters.

Because of perturbations due to noise measurement and modelling errors, the actual force/torque $\tau$ differs from $\tau_{i d m}$ by an error, $e$, such that:

$\tau=\tau_{i d m}+e=\Phi(q, \dot{q}, \ddot{q}) \chi+e$

where:

$\tau=g_{\tau} v_{\tau}$

$v_{\tau}$ is the $(n \times 1)$ vector of the actual current references of the current amplifiers. Equation (12) represents the Inverse Dynamic Identification Model (IDIM).

\section{Identification of the Dynamic Parameters}

The off-line identification of the base dynamic parameters $\chi$ is considered, given measured or estimated off-line data for $\tau$ and $(q, \dot{q}, \ddot{q})$, collected while the robot is tracking some planned trajectories.

$(q, \dot{q}, \ddot{q})$ in (12) are estimated with $(\hat{q}, \hat{\dot{q}}, \hat{\ddot{q}})$, respectively, obtained by band-pass filtering the measure of $q$ [16]. The principle is to sample the identification model (12) at a frequency $f_{m}$ in order to get an over-determined linear system of $\left(n \times n_{m}\right)$ equations and $n_{b}$ unknowns such that:

$Y_{f m}(\tau)=W_{f m}(\hat{q}, \hat{\dot{q}}, \hat{\ddot{q}}) \chi+\rho_{f m}$

In order to cancel the high frequency torque ripple in $Y_{f m}(\tau)$ and to window the identification frequency range into the model dynamics, a parallel decimation procedure low-pass filters in parallel $Y_{f m}$ and each column of $W_{f m}$ and resamples them at a lower rate, keeping one sample over $n_{d}$. This parallel filtering procedure can be carried out with the Matlab decimate function [16]. It is obtained:

$Y(\tau)=W(\hat{q}, \hat{\dot{q}}, \hat{q}) \chi+\rho$

$\rho$ is the (rx1) vector of errors, with $r=n \times n_{m} / n_{d}$, $W(\hat{q}, \hat{\dot{q}}, \hat{\ddot{q}})$ is the $\left(r \times n_{b}\right)$ observation matrix.

In $Y$ and $W$, the equations of each joint $j$ are grouped together such that:

$$
Y=\left[\begin{array}{lll}
\left(Y^{1}\right)^{T} & \ldots & \left(Y^{n}\right)^{T}
\end{array}\right]^{T}, W=\left[\begin{array}{lll}
\left(W^{1}\right)^{T} & \ldots & \left(W^{n}\right)^{T}
\end{array}\right]^{T}
$$

$Y^{j}$ and $W^{j}$ represent the $n_{m} / n_{d}$ equations of joint $j$. The ordinary LS (OLS) solution $\hat{\chi}$ minimizes $\|\rho\|^{2}$.

Using the base parameters and tracking "exciting" reference trajectories, a well conditioned matrix $W$ is obtained. The LS solution $\hat{\chi}$ of (15) is given by:

$\hat{\chi}=\left(\left(W^{T} W\right)^{-1} W^{T}\right) Y=W^{+} Y$

Standard deviations $\sigma_{\hat{\chi}_{i}}$, are estimated assuming that $W$ is a deterministic matrix and $\rho$, is a zero-mean additive independent Gaussian noise, with a covariance matrix $C_{\rho \rho}$, such that:

$C_{\rho \rho}=E\left(\rho \rho^{\mathrm{T}}\right)=\sigma_{\rho}^{2} I_{r}$

$E$ is the expectation operator and $I_{r}$, the $(r \times r)$ identity matrix. An unbiased estimation of the standard deviation $\sigma_{\rho}$ is:

$\hat{\sigma}_{\rho}^{2}=\|Y-W \hat{\chi}\|^{2} /(r-b)$

The covariance matrix of the estimation error is given by: $C_{\hat{\chi} \hat{\chi}}=E\left[(\chi-\hat{\chi})(\chi-\hat{\chi})^{\mathrm{T}}\right]=\hat{\sigma}_{\rho}^{2}\left(W^{\mathrm{T}} W\right)^{-1}$.

$\sigma_{\hat{\chi}_{i}}^{2}=C_{\hat{\chi} \hat{\chi}}(i, i)$ is the $i^{\text {th }}$ diagonal coefficient of $C_{\hat{\chi} \hat{\chi}}$

The relative standard deviation $\% \sigma_{\hat{\chi}_{r i}}$ is given by:

$\% \sigma_{\hat{\chi}_{r i}}=100 \sigma_{\hat{\chi}_{i}} /\left|\hat{\chi}_{i}\right|$, for $\left|\hat{\chi}_{i}\right| \neq 0$

The OLS can be improved by taking into account different standard deviations on joint $j$ equations errors [16]. Each equation of joint $j$ in (15) is weighted with the inverse of the standard deviation of the error calculated from OLS solution of the equations of joint $j$, given by:

$Y^{j}\left(\tau^{j}\right)=W^{j} \chi+\rho^{j}$

This weighting operation normalises the errors in (15) and gives the weighted LS (WLS) estimation of the parameters.

\section{GLOBAL IDENTIFICATION OF THE JOINT DRIVE GAINS}

\section{A. Inverse Dynamic identification Model of the robot and the payload}

The payload is considered as a link $n+1$ fixed to the link $n$ of the robot. The model (11) becomes:

$\tau_{i d m}=\left[\begin{array}{lll}\Phi & \Phi_{u L} & \Phi_{k L}\end{array}\right]\left[\begin{array}{c}\chi \\ \chi_{u L} \\ \chi_{k L}\end{array}\right]$

where:

$\chi_{k L}$ is the $\left(n_{L} \times 1\right)$ vector of the known inertial parameters of the payload;

$\chi_{u L}$ is the $\left(\left(10-n_{L}\right) \times 1\right)$ vector of the unknown inertial 
parameters of the payload,

$\Phi_{k L}$ is the $\left(n \times n_{L}\right)$ jacobian matrix of $\tau_{i d m}$, with respect to the vector $\chi_{k L}$,

$\Phi_{u L}$ is the $\left(n \times\left(10-n_{L}\right)\right)$ jacobian matrix of $\tau_{i d m}$, with respect to the vector $\chi_{u L}$.

The model (12) becomes:

$\tau=\left[\begin{array}{lll}\Phi & \Phi_{u L} & \Phi_{k L}\end{array}\right]\left[\begin{array}{c}\chi \\ \chi_{u L} \\ \chi_{k L}\end{array}\right]+e$

Taking into account that the joint $j$ force/torque depends only on parameters $\chi^{j, n}$ of links $j$ to $n$, the IDIM joint $j$ model is given by:

$\tau^{j}=g_{\tau}^{j} v_{\tau}^{j}=\left[\begin{array}{lll}\Phi^{j, n} & \Phi_{u L}^{j} & \Phi_{k L}^{j}\end{array}\right]\left[\begin{array}{c}\chi^{j, n} \\ \chi_{u L} \\ \chi_{k L}\end{array}\right]+e^{j}$

where $\tau^{j}, \Phi^{j, n}, \Phi_{u L}^{j}, \Phi_{k L}^{j}$, are the respective non zero elements of line $j$ in (24),

$v_{\tau}^{j}$ is the actual joint $j$ current reference given by the numerical controller of the robot.

Equation (25) can be rewritten as:

$v_{\tau}^{j}=\left[\begin{array}{lll}\Phi^{j, n} & \Phi_{u L}^{j} & \Phi_{k L}^{j} \chi_{k L}\end{array}\right]\left[\begin{array}{c}\chi^{j, n} / g_{\tau}^{j} \\ \chi_{u L} / g_{\tau}^{j} \\ 1 / g_{\tau}^{j}\end{array}\right]+e^{j} / g_{\tau}^{j}$

Equation (26) is the minimal model IDIM used to identify the drive gain of joint $j$.

\section{B. Identification of the drive gains}

Considering now that the robot has carried out two trajectories: (a) without the payload and (b) with the payload fixed to the end-effector, the sampling and filtering of the model IDIM (26) can be written as:

$$
\begin{aligned}
V^{j} & =\left[\begin{array}{ccc}
W_{a}^{j} & 0 & 0 \\
W_{b}^{j} & W_{u L}^{j} \chi_{u L} & W_{k L}^{j} \chi_{k L}
\end{array}\right]\left[\begin{array}{c}
\chi^{j, n} / g_{\tau}^{j} \\
\chi_{u L} / g_{\tau}^{j} \\
1 / g_{\tau}^{j}
\end{array}\right]+\rho^{j} / g_{\tau}^{j} \\
& =W_{\chi^{*}}^{j} \chi^{*}+\rho_{j}^{*}
\end{aligned}
$$

where:

$V^{j}$ is the vector of $v_{\tau}^{j}$ samples,

$W_{a}^{j}$ is the observation matrix of joint $j$ in the unloaded case,

$W_{b}^{j}$ is the observation matrix of joint $j$ in the loaded case,

$W_{u L}^{j}$ is the observation matrix for joint $j$ corresponding to the unknown payload inertial parameters,

$W_{k L}^{j}$ is the observation matrix for joint $j$ corresponding to the known payload inertial parameters,

$\hat{\chi}^{*}$ is the weighted LS solution of the over-determined system (27). The last coefficient of vector $\hat{\chi}^{*}$ gives the estimation of the inverse of the joint $j$ drive gain.

Using this global approach, it is possible to only use the knowledge we can have on the payload mass value $M_{L}$ in order to identify the robot drive gains. This mass can generally be accurately measured using a weighting machine. It is assumed that parameter $M_{L}$ appears explicitly in the expression of several joint torques (at least one; if not, the approach cannot be achieved), i.e. $W_{k L}^{j} M_{L} \neq 0$ for all these joints.

For all of them, the drive gains can be identified using the previous approach. Then, using the global identification models for the same joints, other exciting payload inertial parameters (such as the static moments $M X_{L}, M Y_{L}$ or $M Z_{L}$ ) can be estimated and will be used in order to identify the drive gains for the other joints.

The advantage of this method is its simplicity. But its main drawback is that the identification is carried out sequentially and the estimation errors will be cumulated. As a result, it could be preferable to have a complete knowledge of all payload inertial parameters (e.g. using data obtained from CAD software) in order to decouple the drive gain identification for each joint. But, as it will be shown in the next section, the experimental results confirm the efficiency of the proposed approach.

\section{CASE STUdY}

\section{A. Description of the $R X 90$ kinematics}

The Stäubli RX-90 robot (Fig. 1) has a serial structure with six rotational joints. Its kinematics is defined using the modified Denavit and Hartenberg notation (MDH) [10]. In this notation, the link $j$ fixed frame is defined such that the $z_{j}$ axis is taken along joint $j$ axis and the $x_{j}$ axis is along the common normal between $z_{j}$ and $z_{j+1}$ (Fig. 1). The geometric parameters defining the robot frames are given in Table 1. The payload is denoted as the link 7 . The parameter $\sigma_{j}=0$, means that joint $j$ is rotational, $\alpha_{j}$ and $d_{j}$ parameterize the angle and distance between $z_{j-1}$ and $z_{j}$ along $x_{j-1}$, respectively, whereas $\theta_{j}$ and $r_{j}$ parameterize the angle and distance between $x_{j-1}$ and $x_{j}$ along $z_{j}$, respectively. For link 7, $\sigma_{j}=2$ means that the link 7 is fixed on the link 6. Since all the joints are rotational then $\theta_{j}$ is the position variable of joint $j$. It should be mentioned that the variables of joints 2 and 3 in the present notation are obtained from the measured values of joints 2 and 3 using the relations, $\theta_{2}=\theta_{2 s t a u b l i}-\pi / 2, \theta_{3}=\theta_{3 \text { staubli }}+\pi / 2$. The main advantage of using the MDH notation is that the identifiable inertial parameters can be determined 


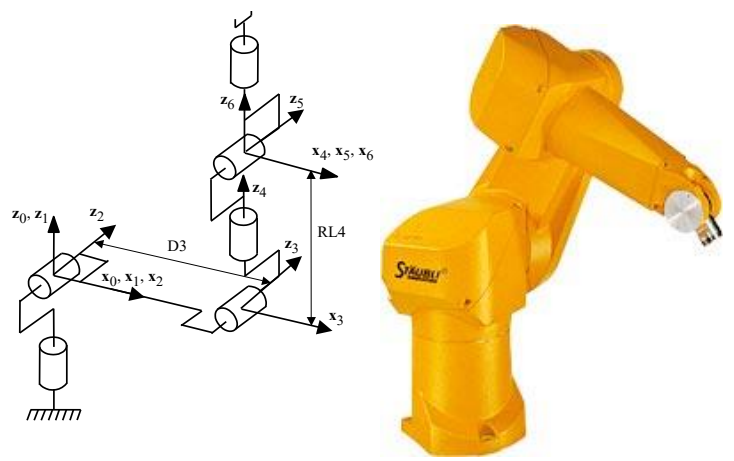

Fig. 1. Link frames of the RX-90 robot

TABLE I

\begin{tabular}{ccclcll}
\multicolumn{6}{c}{ GEOMETRIC PARAMETERS OF THE RX-90 ROBOT WITH THE PAYLOAD } \\
\hline \hline$j$ & $\sigma_{j}$ & $\alpha_{j}$ & \multicolumn{1}{c}{$d_{j}$} & $\theta_{j}$ & $r_{j}$ \\
\hline 1 & 0 & 0 & 0 & $\theta_{1}$ & 0 \\
2 & 0 & $-\pi / 2$ & 0 & $\theta_{2}$ & 0 \\
3 & 0 & 0 & $D_{3}=0.45 \mathrm{~m}$ & $\theta_{3}$ & 0 \\
4 & 0 & $+\pi / 2$ & 0 & $\theta_{4}$ & $R L_{4}=0.45 \mathrm{~m}$ \\
5 & 0 & $-\pi / 2$ & 0 & $\theta_{5}$ & 0 \\
6 & 0 & $+\pi / 2$ & 0 & $\theta_{6}$ & 0 \\
7 & 2 & 0 & 0 & 0 & 0 \\
\hline \hline
\end{tabular}

symbolically using simple closed-form rules [3], [6], [11] and it is possible to generalize the proposed methods for tree structure robots and closed loop robots [5], [6], [12], [13].

\section{B. Identification of the drive gains}

The method is validated using a calibrated payload (Fig. 2). Its mass has been measured with a weighting machine $\left(M_{L}=7.025 \mathrm{Kg} \pm 0.050 \mathrm{Kg}\right)$. The other parameters have been estimated using CAD software. They are given in table 2. Their values are accurate due to the simplicity of the payload shape (Fig. 2).

For the RX 90, it can be shown that:

- the torques of joints 1 to 3 depends on all payload parameters;

- $\quad$ the torques of joints 4 and 5 do not depend on parameter $M_{L}$;

- the torques values of joint 6 do not depend on parameters $M_{L}$ and $M Z_{L}$.

Moreover, our experiments have shown that parameters $M Z_{L}, X X_{L}, Y Y_{L}$ and $Z Z_{L}$ are too small to allow the correct calibration of the drive gains of joint 5 and 6 .

Therefore, the drive gains of joints 1 to 4 only are identified. In order to identify the other drive gains, more exciting payload should be designed, especially with large values for parameters $M X_{L}$ and $M Y_{L}$.

Staübli's control software provides directly the value of the joint torque. Therefore, it is proceeded to a calibration of the drive gains compared with a priori values used in the controller which are taken equal to 1 . A first identification of both the RX 90 dynamic parameters and the load inertial parameters is achieved using the IDIM-LS method with the model (25), as detailed in [17] (case 1). The results are presented in table 2. It can be observed that the mass $M_{L}$

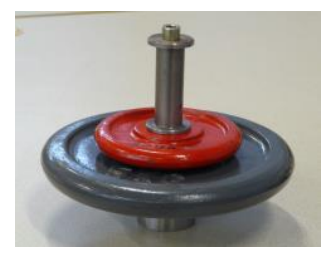

Fig. 2. The $7.025 \mathrm{Kg}$ payload

TABLE 2

IDENTIFICATION OF THE PAYLOAD DYNAMIC PARAMETERS

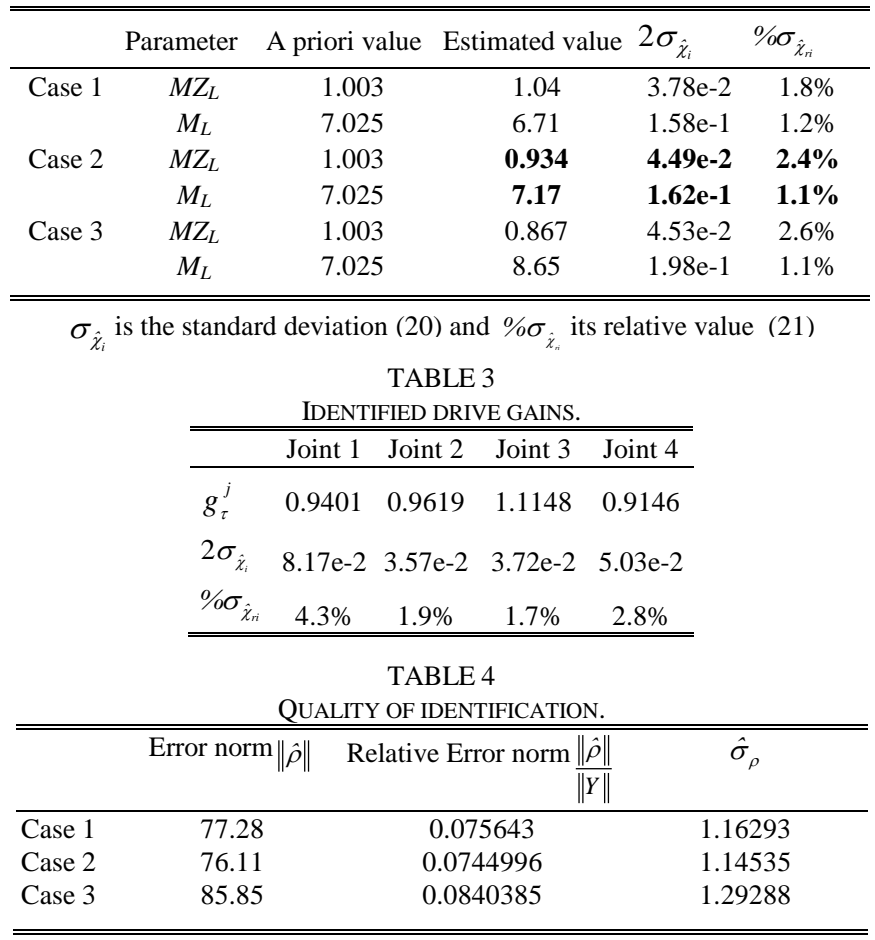

$\|\hat{\rho}\|=\|Y-W \hat{\chi}\|$ is the minimal norm of error, $\hat{\sigma}_{\rho}$ is given by (19).

and the first moment $M Z_{L}$ are poorly identified with the manufacturer's gains.

Then, the new drive gains for joints 1 to 4 are identified using our new approach (case 2). The identified drive gains are presented in table 3 . It can be shown that there is an average of $8 \%$ error with respect to the a priori drive gains.

A new identification of the payload dynamic parameters is achieved with the identified gains. The results are presented in table 2. It can be observed that the mass $M_{L}$ and the first moment $M Z_{L}$ are better identified using the new gains.

In table 4 , the quality of identification for the two studied cases in terms of reduction of the norm of the error vector $\rho$ is shown. With the identified gains, the quality of identification is largely improved.

Figure 3 shows that the reconstructed torques with the identified gains for joints 1 to 4 are close to the actual ones.

In order to show the sensitivity of the identification results to the variation of the drive gain values, a $20 \%$ error on these gains (with respect to those identified in case 2) is simulated (case 3). The quality of identification is clearly worst in this case (table 4). The identified payload parameters are given in table 2 . Their values are far from reality. 

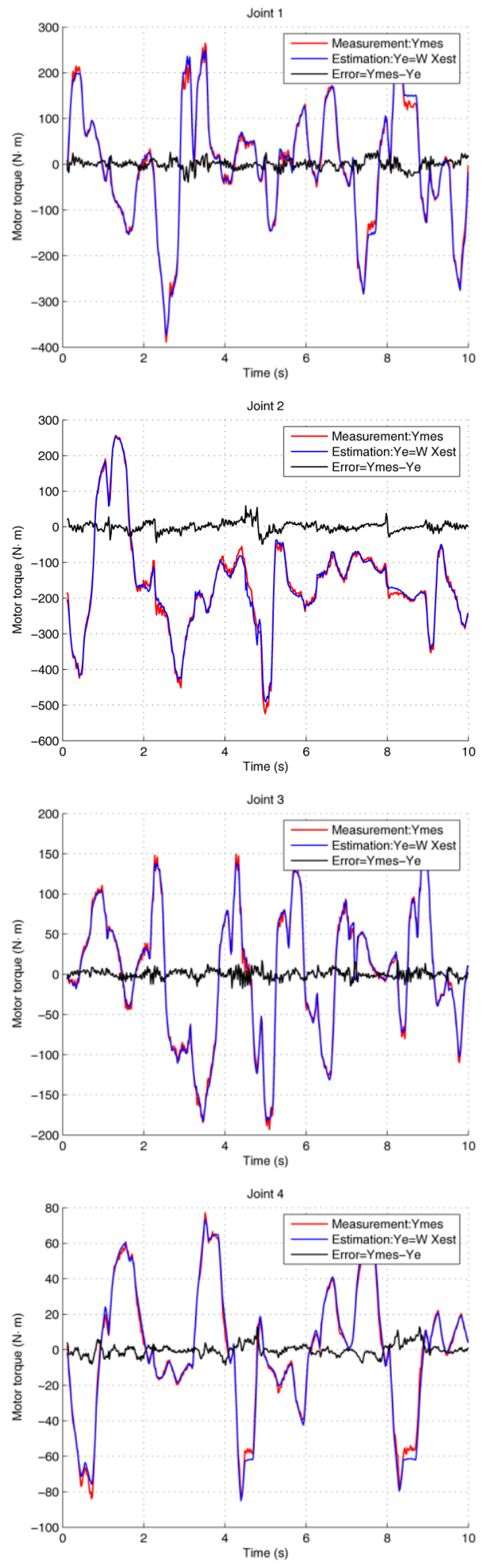

Fig.3. Torque validation with identified drive gains, Case 2.

Finally, in order to validate the new drive gain values, a new payload of $4.275 \mathrm{Kg} \pm 0.050 \mathrm{Kg}$, is identified. The identified mass is $M_{L}=4.35 \mathrm{Kg}$, with $2 \sigma_{\hat{x}_{i}}=0.150$, which is very close to the exact value. It is possible to conclude that the drive gains have been well identified.

\section{CONCLUSION}

This paper has presented a new method for the identification of the total drive gains for robot joints in one step. This is a method easy to implement which does not need any special test or measurement on elements inside the joint drive train. It is based on a IDIM-LS technique using current reference and position sampled data while the robot is tracking one reference trajectory without load fixed on the robot and one trajectory with a known payload fixed on the robot, whose inertial parameters are measured or calculated by a CAD model. The method has been experimentally validated on a Stäubli RX-90 robot. With the new identified drive gains, the identification of the total dynamic model of the robot has been improved and another payload has been accurately identified. This shows the effectiveness of the method.

\section{REFERENCES}

[1] C. Canudas de Wit and A. Aubin, "Parameters identification of robots manipulators via sequential hybrid estimation algorithms", Proc. IFAC Congress, Tallin, 1990, pp. 178-183.

[2] M. Gautier, "Identification of robots dynamics", Proc. IFAC Symp on Theory of Robots, Vienne, Austria, December 1986, p. 351-356.

[3] M. Gautier and W. Khalil, "Direct calculation of minimum set of inertial parameters of serial robots", IEEE Trans. on Robotics and Automation, Vol. RA-6(3), 1990, p. 368-373.

[4] M. Gautier, W. Khalil and P.P. Restrepo, "Identification of the dynamic parameters of a closed loop robot", Proc. IEEE Int. Conf. on Robotics and Automation, Nagoya, may 1995, p. 3045-3050.

[5] J. Hollerbach, W. Khalil, M. Gautier, "Model Identification", chapter 14 in Siciliano Bruno, and Oussama Khatib. eds "Springer Handbook of Robotics », Springer, 2008.

[6] W. Khalil and E. Dombre, "Modeling, identification and control of robots", Hermes Penton London, 2002.

[7] P.K. Khosla and T. Kanade, "Parameter identification of robot dynamics", Proc. $24^{\text {th }}$ IEEE Conf. on Decision and Control, FortLauderdale, déc. 1985 , p. 1754-1760.

[8] Z. Lu, K.B. Shimoga and A. Goldenberg, "Experimental determination of dynamic parameters of robotic arms", Journal of Robotics Systems, Vol. 10, Nº, 1993, p.1009-1029.

[9] P.P. Restrepo and M. Gautier, "Calibration of drive chain of robot joints," Proceedings of the 4th IEEE Conference on Control Applications, 1995, pp. 526-531.

[10] W. Khalil and J.F. Kleinfinger, "A new geometric notation for open and closed loop robots", Proceedings of the IEEE International Conference on Robotics and Automation, 1986, San Francisco.

[11] W. Khalil and F. Bennis, "Symbolic calculation of the base inertial parameters of closed-loop robots", The Int. J. of Robotics Research, Vol. 14(2), avril 1995, p. 112-128.

[12] R. Featherstone, D.E. Orin, "Dynamics", chapter 2 in Siciliano Bruno, and Oussama Khatib. eds « Springer Handbook of Robotics », Springer, 2008.

[13] M. Gautier, "Numerical calculation of the base inertial parameters", Journal of Robotics Systems, Vol. 8, №4, 1991, pp. 485-506.

[14] W. Khalil, D. Creusot, "SYMORO+: A system for the symbolic modelling of robots", Robotica, Vol.15, 1997, p.153-161.

[15] H. Mayeda, K. Yoshida and K. Osuka, "Base parameters of manipulator dynamic models", IEEE Trans. on Robotics and Automation, Vol. RA-6(3), 1990, p. 312-321.

[16] M. Gautier, "Dynamic identification of robots with power model", Proceedings of the IEEE International Conference on Robotics and Automation, 1997, Albuquerque, New Mexico, April, pp. 1922-1927.

[17] W. Khalil, M. Gautier and P. Lemoine, "Identification of the payload inertial parameters of industrial manipulators," Proceedings of the IEEE International Conference on Robotics and Automation, 2007, Roma, Italy, April 10-14, pp. 4943-4948. 